\title{
Influenza drug could abort a pandemic
}

\section{It should be taken, not pre-emptively, but after infection is revealed by a rapid flu test.}

Sir - An influenza pandemic will occur at some time in the future: having worked on flu viruses since 1959, I am certain of this. If the deadly H5N1 'bird flu' suddenly acquired human transmissibility, while retaining pathogenicity, the resulting pandemic would cause millions of human deaths. If the pandemic were caused by another bird influenza virus, or if the human H2 virus that disappeared in 1968 were to return, humanity would still be in for a bad time.

No 'pandemic vaccine' could be stockpiled, because of uncertainty about the virus strain. So what can be done? School closure, quarantine, travel restrictions and so on are unlikely to be more effective than a garden hose in a forest fire.

There are, however, two safe antiviral drugs that are effective against all flu viruses, including H5N1. These are the neuraminidase-inhibitors zanamivir/ Relenza and oseltamivir/Tamiflu. (Although my crystallization of flu virus neuraminidase led to the development of these drugs and I have a financial interest in Relenza, I have none in Tamiflu.)
Tamiflu is a small carbocyclic molecule that was rationally designed from a knowledge of the X-ray crystal structure of influenza virus neuraminidase. The virus needs neuraminidase to escape from infected cells and spread through the body. The catalytic site of flu neuraminidase, unlike the variable surface antigens, is conserved by all strains of the virus. Tamiflu was designed to fit precisely in the catalytic site of the enzyme, inhibiting its activity.

To be effective, Tamiflu has to be given before infection, or very soon after flu symptoms appear. The time needed to obtain a prescription is a serious drawback.

Although governments around the world are reported to be stockpiling Tamiflu, their strategies for using it are not clear. Britain is reported to have 14.6 million doses of Tamiflu, enough for a quarter of the population. Australia is reported to have enough to protect 200,000 front-line workers prophylactically during a pandemic: two pills a day for 50 days.

This strategy, I believe, is wrong. A more efficient use would be to have Tamiflu available over the counter in local pharmacies, coupled with a rapid, sensitive and accurate flu diagnostic test. People with flu symptoms could then go immediately to the pharmacy, be rapidly tested to see if the infection is influenza and, if it is positive, be given Tamiflu.

Not only would the infection be curtailed - the person would, on recovery, be immune to reinfection with the same virus.

This procedure could be called 'aborted-infection immunization' and should be used in the early stages of a pandemic, when no vaccine is available, or in the inter-pandemic period by those people who do not take the vaccine or who experience vaccine failure.

The neuraminidase inhibitors exist. They do work. Correct use could be achieved through public education. In a pandemic they would alleviate much of the flu victims' misery, reduce economic losses and probably prevent deaths.

\section{Graeme Laver}

Barton Highway, Murrumbateman,

New South Wales 2582, Australia

graeme.laver@bigpond.com

\section{Changes in China call for new health solutions}

Sir - The Commentary article "Lessons from the past" by Z. Dong and colleagues (Nature 433, 573-574; 2005), on China's public-health system, has touched upon an urgent issue that may influence the course of China's future. Their attempt to apply such lessons in today's China, however, may require a deeper look at the fundamental changes that have taken place in the country from one era to the other.

The "glorious beginning" under Mao's regime might have been due more to totalitarian control, with communities isolated and movement restricted, than to the health-care system at that time. Although that may have been more fair than today's system, it was at least as inadequate.

The serious medical problems facing today's more open and free China did not have the chance to flourish during that earlier period. Contrast the early 1980s, for example, when sexually transmitted diseases were rare, with the present, when HIV/AIDS has reached epidemic status in parts of the country.

Prevention is the key to public health. But remedies from the past should not be relied on to solve a crisis today, when one is confronted with totally different problems in a totally different society. Yonghong Li Celera Diagnostics, 1401 Harbor Bay Parkway, Alameda, California 94502, USA

\section{NIH conflicts rules are not right for universities}

Sir — We agree with your Editorial "Taking a hard line on conflicts" (Nature 433, 557; 2005) that "scientists and institutions everywhere should be sure that their own houses are fully in order". But the academic research community and the National Institutes of Health (NIH) have different societal roles, expectations and regulatory histories. These differences are essential to understanding why conflict-of-interest policies that apply to researchers employed by the NIH should not be extended to the $\mathrm{NIH}$-funded university community.

Universities' long-standing interactions with industry have produced enormous benefits. Still, the academic community recognizes that the US public's support of academic, and especially biomedical, research depends on maintaining their confidence and trust.

Research universities have had policies on faculty consulting with industry for decades and have gained deep experience in regulating conflicts of interest across all disciplines. Theyhavegiven specialattention to conflicts in biomedical research since mandatory federal regulations for NIHfunded academics were published in 1995, enhanced by federal guidelines in 2004.

Academia has used these regulations as the base on which to build more robust standards, such as those on human research set out by the Association of American Medical Colleges (AAMC; see www.aamc. org/members/coitf/start.htm). Our 2004 survey of medical schools (see Nature 431, 725 ; 2004) indicated that most have adopted all or substantial portions of the AAMC recommendations, thereby going well beyond federal requirements.

However stringent the standards and supervision, violations will occur. But to react with overzealous regulations would inhibit useful partnerships and the social benefits that flow from them. As long as universities and medical schools continue to take seriously the enforcement of strict standards, relationships with industry can be principled, protective of research participants and scientific integrity and remain capable of withstanding intense public scrutiny.

David Korn, Susan H. Ehringhaus

Association of American Medical Colleges, 2450

North Street NW, Washington DC 20037, USA 